\title{
Characterization of the polishing-induced contamination of fused silica optics
}

\author{
Mathilde Pfiffer $^{1}$ | Jean-Louis Longuet ${ }^{2}$ | Christine Labrugère ${ }^{3}$ | Evelyne Fargin ${ }^{4}$ | \\ Bruno Bousquet $^{5}$ | Marc Dussauze ${ }^{6}$ | Sébastien Lambert ${ }^{2}$ | Philippe Cormont ${ }^{1}$ | \\ Jérôme Néauport ${ }^{1}$
}

${ }^{1}$ Commissariat à l'Energie Atomique et aux Energies Alternatives, Centre d'Etudes

Scientifiques et Techniques d'Aquitaine,

Le Barp, France

${ }^{2}$ Commissariat à l'Energie Atomique et aux Energies Alternatives, Le Ripault, Monts,

France

${ }^{3}$ Plateforme Aquitaine de Caractérisation des Matériaux, UMS3626, Pessac, France

${ }^{4}$ Institut de Chimie de la Matière Condensée de Bordeaux, Pessac, France

${ }^{5}$ Centre d'Etudes des Laser Intenses et Applications, UMR 5107 CNRS, Université de Bordeaux, CEA, Talence, France

${ }^{6}$ Institut des Sciences Moléculaires, UMR 5255 CNRS, Talence, France

\section{Correspondence}

Mathilde Pfiffer, Commissariat à l'Energie Atomique et aux Energies Alternatives, Centre d'Etudes Scientifiques et Techniques

d'Aquitaine, Le Barp, France.

Email: mathilde.pfiffer@cea.fr

\begin{abstract}
In this work, the polishing-induced contamination layer at the fused silica optics surface was studied with various interface analysis techniques: Secondary Ion Mass Spectroscopy (SIMS), Electron Probe Microanalysis (EPMA), X-Ray Photoelectron Spectroscopy (XPS), and Inductively Coupled Plasma-Optical Emission Spectroscopy (ICP-OES). Samples were prepared using an MRF polishing machine and cerium-based slurry. The cerium and iron penetration and concentration were measured in the surface out of defects. Cerium is embedded at the surface in a $60 \mathrm{~nm}$ layer and concentrated at $1200 \mathrm{ppmw}$ in this layer while iron concentration falls down at $30 \mathrm{~nm}$. Spatial distribution and homogeneity of the pollution were also studied in the scratches and bevel using SIMS and EPMA techniques. We saw evidence that surface defects, such as scratches, are specific places that hold the pollutants. This overconcentration is also observed in the chamfer. These new insights into the polishing-induced contamination of fused silica optics and it repartition have been obtained using various characterization methods. Advantages and disadvantages of each one are discussed.
\end{abstract}

\section{K E Y W O R D S}

fused silica, impurities, polishing, spectroscopy

\section{1 | INTRODUCTION}

Glass manufacturing includes a large number of specific processes that range from the shaping of raw materials through grinding to polishing. Among them, the polishing step is a particularly complex process because it is a chemicomechanical process dependent on the nature of optical material and the type of polishing compound. One of the most specific features concerning glass polishing is the formation of a thin layer on the surface which is due to a chemical reaction between the surface and the polishing slurry. This modified surface layer is generally called Beilby layer. This name was firstly attributed to polished metal surface layer; however, it is now used for all polished layers. ${ }^{1}$ Trogolo et al. ${ }^{2}$ highlighted the silica structure modification near the surface and estimated its depth between 100 and $200 \mathrm{~nm}$. These chemical reactions between the surface and the slurry are responsible for the contamination of the surface by impurities initially contained in the slurry. Bach $^{3}$ then Suratwala et al. ${ }^{4}$ analyzed in details the reactions between silica surface and cerium, which is an active element in the polishing process. The chemical mechanism starts with the condensation reaction between the $\mathrm{Si}-\mathrm{OH}$ silica surface and $\mathrm{Ce}-\mathrm{OH}$ ceria particle surface. The subsequent reactions are hydrolysis of $\mathrm{Si}-\mathrm{O}$ $\mathrm{Si}$ and $\mathrm{Ce}-\mathrm{O}-\mathrm{Ce}$ bonds. Cerium penetrates in the silica surface because of the hydrolysis of the $\mathrm{Ce}-\mathrm{O}-\mathrm{Ce}$ bonds. Other species, such as potassium $\mathrm{K}$, penetrate by diffusion 
into the surface during the polishing process. Considering the major role of impurities in the optical properties, the surface layer induced by polishing process has been the subject of major researches for 30 years.,

Recently, high-power laser optics production provided a renewed interest in the polish-induced layer. ${ }^{6,7}$ Indeed, it is well-known that such impurities can absorb ultraviolet light and are in some cases responsible for laser-induced damage in the ultraviolet $(351 \mathrm{~nm}) .{ }^{8}$ In 1998, Kozlowski et al. detected a metallic pollution at the fused silica polished surface and measured an exponential decay of the contaminants concentration. ${ }^{6}$ In the same year, Camp et al. ${ }^{9}$ observed a "gray haze" damage morphology and linked it with the cerium polishing-induced contamination because cerium presents a large absorption cross section at $351 \mathrm{~nm}$ compared to the other elements identified in the polishinginduced layer. ${ }^{6}$ It is also the case for iron that can penetrate the polished layer by Magneto-Rheological Finishing (MRF) polishing or earlier grinding steps. For that reason, recent studies mainly focus on cerium and iron depth profiles. Pollution depth and concentration values available in this relatively large literature are quite dispersed. This is illustrated in Table 1 where we summarized depth and concentration values of cerium and/or iron with the corresponding characterization method used to establish these results. The polishing process used to manufacture the sample is also detailed (conventional corresponds to loose abrasive polishing on pad or pitch polisher).

Table 1 shows that the concentration and depth penetration of contaminants are reported to be significantly different. Such discrepancies can be explained by two factors. First, it has been shown that the surface layer modification is dependent on the processing parameters applied. Suratwala et al. ${ }^{4}$ and Wang et al. ${ }^{17}$ explained that the cerium depth penetration is consistent with a mechanism which depends on the relative rate of hydrolyze between $\mathrm{Ce}-\mathrm{O}-$ $\mathrm{Ce}$ and $\mathrm{Si}-\mathrm{O}-\mathrm{Si}$ bonds, where the ratio increases with the interface temperature which increases with polishing removal rate. However, the process parameters cannot totally explain the huge differences in depth and concentration.

The second factor which can induce differences is the type of characterization method used to quantify pollution depth and concentration. The most conventional technique is the SIMS, which uses an ions beam to sputter the surface then the particles emitted from the sample are detected with a magnetic mass spectrometer or a time-of-flight spectrometer. Gao et al., ${ }^{13}$ Neauport et al., ${ }^{7}$ and Catrin et al. ${ }^{14}$

TABLE 1 A review of publications related to cerium and/or iron depth penetration and concentration in the fused silica surface polished with a CeO slurry

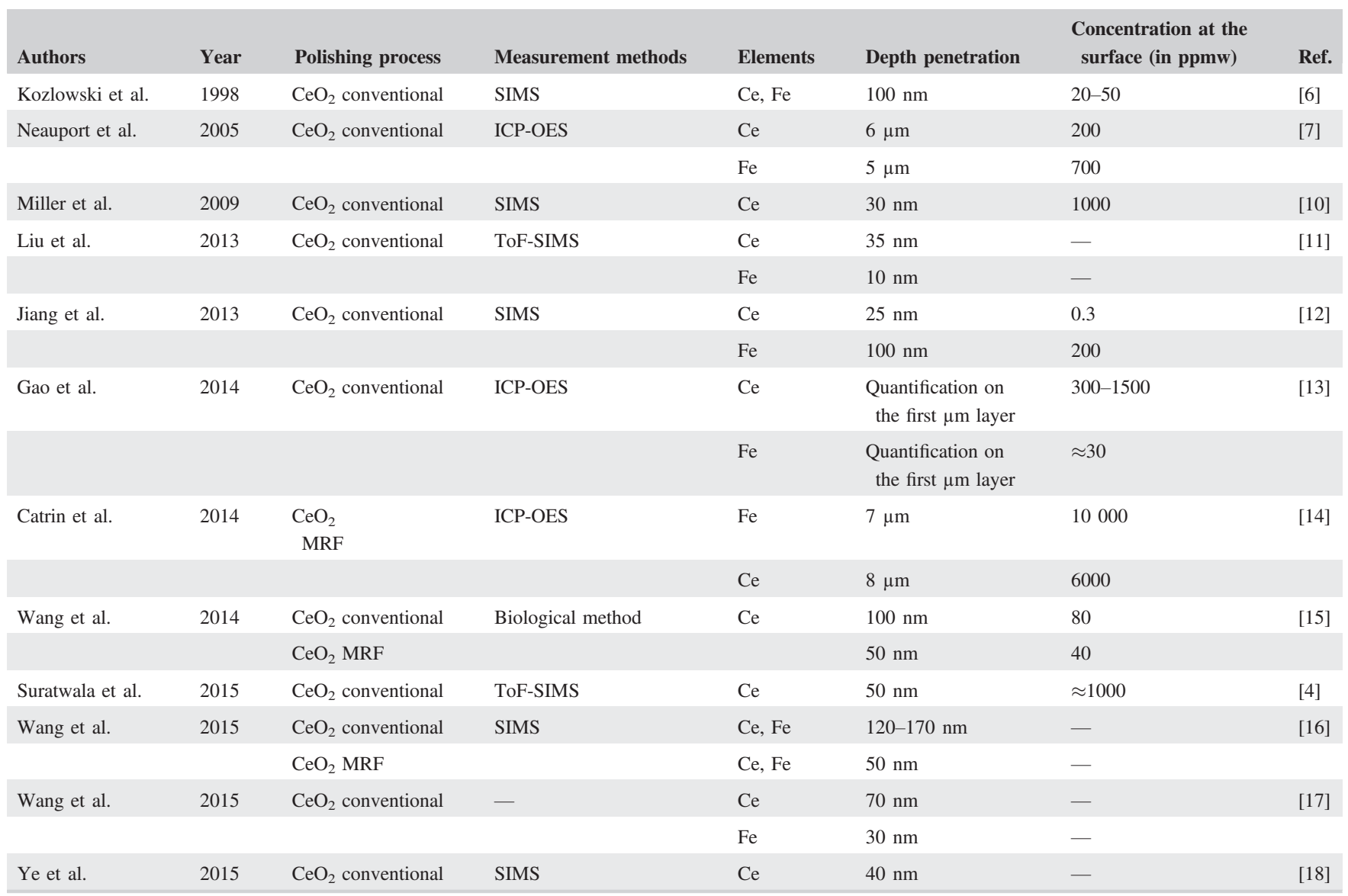


proposed to use the ICP-OES technique. An ICP-OES analysis starts with the surface ionization by plasma then the surface composition is determined by an optical spectrometer when the particles relax and emit at a specific wavelength. Table 1 highlights the analysis method contribution on the results dispersion. SIMS measurements show a maximum pollution depth penetration of $200 \mathrm{~nm}$ associated with concentration values ranging between 0.3 and 1000 ppmw. Unlike SIMS measurements, the ICP-OES measurements evidence that cerium goes deeply into the surface, ie, a few micrometers and impurities concentrations are also higher than the ones obtained with SIMS, namely, 100 to 10.000 ppmw. Finally, Table 1 seems to confirm that these impurities-sensitive measurements appear to be also highly dependent on the technique used.

The aim of this study is mainly to get a better knowledge of contamination depth penetration and concentration on a fused silica polished surface by using various characterization methods and to point out that they bring complementary information on the interface composition as it has been already done on metallic materials and thin films. ${ }^{19,20}$ For this purpose, samples were prepared using a MRF polishing machine and a cerium-based slurry so characterizations focused on cerium and iron contamination. We used MRF polishing to add an iron contamination. Polished surfaces were characterized using SIMS, ICP-OES, XPS, and EPMA with experimental protocols detailed in Section 2. We will then discuss results in Section 3 with an emphasis on surface and depth analysis, as well as homogeneity of pollution on the surface. We will then bring our conclusions.

\section{EXPERIMENTAL SETUP}

\section{1 | Samples preparation}

Samples were circular with a diameter of $50 \mathrm{~mm}$ and their chamfers were $1 \mathrm{~mm}$ large with an angle of $45^{\circ}$. All samples were prepared with the same protocol to study the influence of the technique on the measurements. A set of fused silica samples (Corning Si7980 fused silica blank, $50 \mathrm{~mm}$ diameter $\times 5 \mathrm{~mm}$ thick) were MRF polished on a QED Q-22 XE machine with a 50-mm-diameter wheel by using a standard $\mathrm{C} 10+$ fluid containing cerium oxide particles. $^{21}$ Polishing parameters were set to remove a $1-\mu \mathrm{m}-$ thick layer. The following parameters were kept constant for all samples: wheel speed of $600 \mathrm{rpm}$, pump speed of $80 \mathrm{rpm}$, and a volumetric removal rate of $0.0273 \mathrm{~mm}^{3} / \mathrm{min}$. After polishing, samples were cleaned in a $50^{\circ} \mathrm{C}$ deionized water bath with RBS50 detergent and $68 \mathrm{kHz}$ ultrasonic for 6 minute. Following leaching, optics were rinsed in a deionized bath during 6 minute then dried vertically.

\subsection{TEM characterization of the polishing fluid}

Transmission Electron Microscopy (TEM) was performed to analyze the morphology of particles in the polishing fluid. As the polishing fluid is a powder mixed with deionized water, this TEM analysis started with an observation of the powder and then the mixture between this powder and water was studied.

\section{3 | SEM characterization}

Scanning Electron Microscopy (SEM) was accomplished on a MRF polished fused silica sample surface using a JSM 6700 (JEOL, Tokyo, Japan) operating at $8 \mathrm{keV}$. Sample was coated with a gold $5 \mathrm{~nm}$ layer prior to analysis.

\section{4 | SIMS measurements}

SIMS depth measurements were performed using a TOFSIMS5 from IONTOF Company (Münster, Germany) with a $\mathrm{Bi}^{+}$primary ion beam operated at $25 \mathrm{keV}$ and an $\mathrm{O}_{2}$ sputtering ion beam. A $100 \mu \mathrm{m} \times 100 \mu \mathrm{m}$ analysis area was used for measurements performed at the surface, in the scratch and in the chamfer. No calibration was performed because the relative measurements were enough to get the depth penetration. Quantification is obtained by alternative techniques.

\subsection{EPMA measurements}

Electron Probe Microanalysis (EPMA) was done with a CAMECA SX50 (Cameca, Gennevilliers, France) microprobe, equipped with four wavelength dispersive spectrometers. Two types of measurements were performed: a quantitative analysis on the surface and a qualitative mapping to study the spatial distribution at the edge of the sample, between surface and chamfer. For both types of measurement, Cerium $L_{\text {alpha }}$ line $(4.84 \mathrm{keV})$ and Iron $K_{\text {alpha }}$ line $(6.40 \mathrm{keV})$ were simultaneously analyzed with a pentaerythriol crystal for cerium and a lithium fluoride (LiF) crystal for iron.

Specific parameters were applied to study the spatial distribution of cerium and iron. Qualitative X-Ray maps of $640 \mu \mathrm{m} \times 640 \mu \mathrm{m}$ at the edge of the sample, showing both surface and chamfer, were performed with a $15 \mathrm{kV}$, $200 \mathrm{nA}$, and $5 \mu \mathrm{m}$ defocused beam with $100 \mathrm{~ms}$ dwell time.

For quantitative measurements, specific Thin Film Analysis (TFA) program was used. ${ }^{22}$ Specific parameters listed above were employed. A line of $1200 \mu \mathrm{m}$ length was analyzed using a defocused beam of $5 \mu \mathrm{m}$ in 
diameter and $10 \mu \mathrm{m}$ steps with stage motion mode. High beam intensities (up to $300 \mathrm{nA}$ ) and long dwell time counting (around 10 minute per point) were used to maximize sensitivity. Low Ce-doped glass and pure iron (standards block layout no. 4965 from Agar Scientific ${ }^{\circledR}$, Stansted, UK) were used as standards for quantitative calibration routine.

\section{6 | XPS measurements}

XPS measurements were performed on a K-ALPHA spectrometer (Thermofisher Scientific, Waltham, MA) using a monochromatic $\mathrm{Al} K \alpha_{1,2} \mathrm{X}$-ray source and a $400 \mu \mathrm{m}$ spot diameter. The residual pressure in the analysis chamber was less than $4.10^{-7}$ mbar. An electronic charge compensation has been used because the fused silica sample was insulating. To obtain a depth profile, a 1-mm-diameter $\mathrm{Ar}^{+}$ beam working at $500 \mathrm{eV}$ was used.

\section{7 | ICP-OES measurements}

The method consists in dissolving a layer of a silica polished sample in $\mathrm{HF} / \mathrm{HNO}_{3}$ solution and then analyzing this solution by ICP-OES to have access to the quantity of each pollutant embedded in this layer. Thickness layer is measured by mass losses. This sample digestion was repeated several times to obtain asymptotic impurities concentrations. Complete details of the analytical procedure can be found elsewhere. ${ }^{7}$ This technique, in contrast with SIMS, EPMA, or XPS, characterizes the polishinginduced contamination of a full sample, ie, including chamfer.

\section{3 | RESULTS AND DISCUSSION}

\section{1 | Polishing fluid characterization}

Surface contamination is mostly introduced by the polishing fluid, so we characterized it to improve our knowledge of the morphology of particles which can be embedded in the surface after the polishing process. We previously determined the MRF polishing fluid composition by ICPOES and we concluded that cerium oxide and iron represent more than $90 \%$ of the fluid composition. TEM observations were performed on the powder before mixing it with the fluid carrier (Figure 1A) and on the mixed fluid (Figure 1B).

These images illustrate that iron particles are circular with a diameter of around $1 \mu \mathrm{m}$, whereas cerium oxide particles are much smaller with substructures in the $200 \mathrm{~nm}$ range. After mixing powder with water, cerium oxide particles aggregate around iron particles as can be seen in Figure 1B.

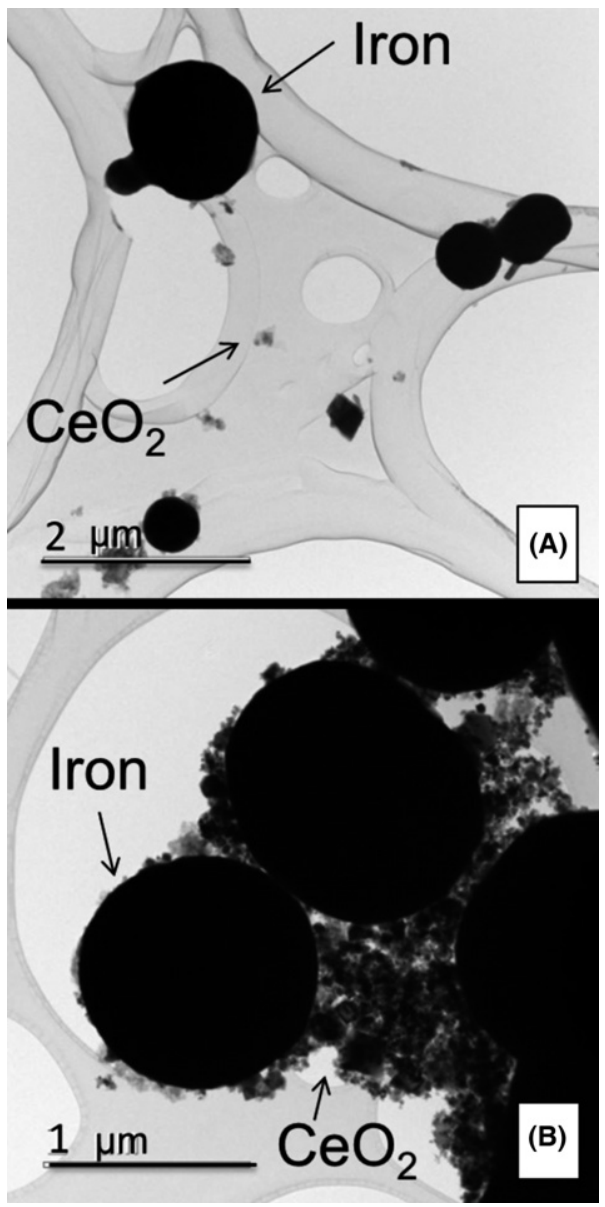

F I G U RE 1 TEM characterization of MRF polishing fluid: (A) powder phase and (B) fluid after mixing between powder phase and deionized water

\section{2 | Surface analysis and contamination depth profiles}

\subsection{1 | SIMS results}

Measurements on a MRF polished surface were carried out on three points located in the center and the middle of the radius of a 50-mm-diameter sample. These measurements reveal the presence of alkali and metal elements on the extreme surface. Figure 2 displays typical SIMS profiles for cerium and iron contamination.

The cerium is concentrated at the surface and disappears under $60 \mathrm{~nm}$. This result is consistent with Suratwala's 4 depth profile measurements. For iron, the concentration falls off after $30 \mathrm{~nm}$. The asymmetry between cerium and iron penetration depths can be explained by the different particles sizes. As mentioned in paragraph 3.1, cerium particles are five times smaller than iron particles. Moreover, as the three plots are superimposed for both cerium and iron, we conclude that the contamination is uniform between the three points of measurement. These three points of measurement have been randomly chosen, 


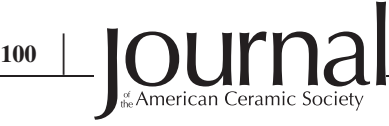

Cerium

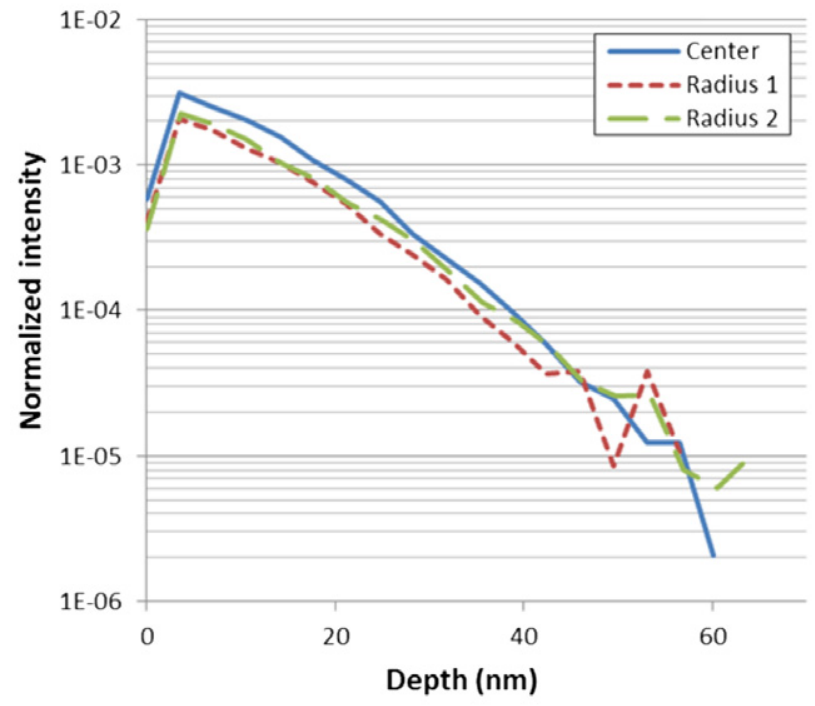

Iron

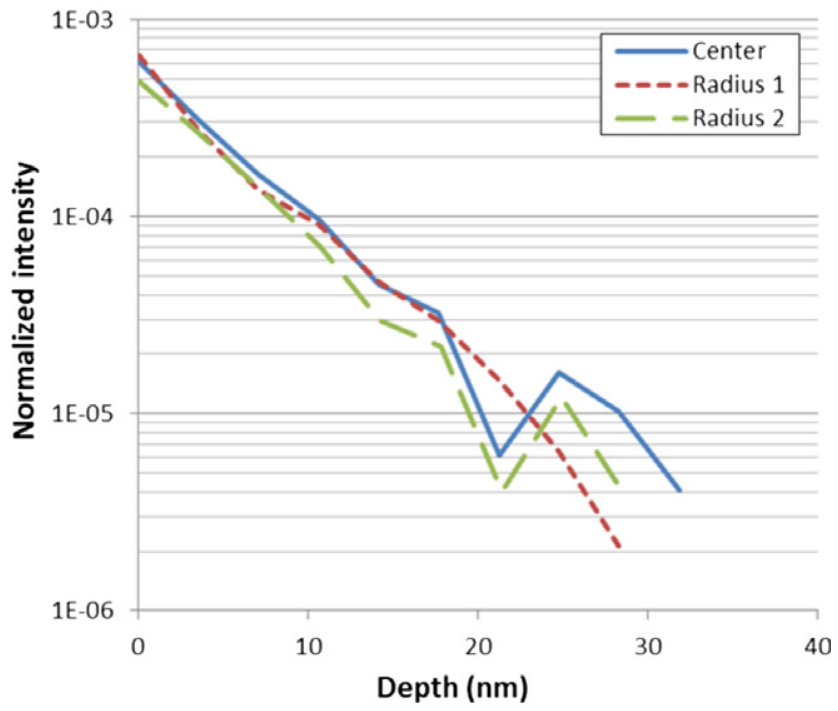

F I G U RE 2 SIMS generated depth profiles of cerium (A) and iron (B) on the same MRF polished fused silica sample at different places on the surface [Color figure can be viewed at wileyonlinelibrary.com]

consequently cerium and iron contaminations are homogeneous at the polished surface.

\subsection{2 | EPMA results}

To identify and quantify the impurities in the polished layer, a microprobe analysis was carried out. Despite high beam intensities and long dwell time counting were used, iron remained under detection limits. The study then only focused on cerium.

As contamination induced by polishing is on surface, the Beilby layer was analyzed with a specific EPMA TFA procedure. In this setup, the sample is considered as a thin top layer of cerium-doped fused silica glass deposited on a substrate made of pure fused silica glass. In general, multiple high voltage (HV) beam accelerations are used to scan more or less the top layer during TFA analysis. Assuming the thickness determined by SIMS analysis, 8 and $15 \mathrm{kV}$ were chosen to have the best sensitivity for TFA analysis on this sample. These high voltage beam accelerations were chosen doing a compromise between the sensitivity of the lines we focused on (Cerium $\mathrm{L}_{\text {alpha }}$ line $=4.84 \mathrm{keV}$ and Iron $\mathrm{K}_{\text {alpha }}$ line $=6.40 \mathrm{keV}$ ) and the analyzed layer depth. A line of more than $1 \mathrm{~mm}$ length on the sample surface was analyzed using a defocused beam of $5 \mu \mathrm{m}$ in diameter and $10 \mu \mathrm{m}$ steps with stage motion mode.

The ratio between the intensity of $\mathrm{Ce}$ measured in the sample (Ix) over the one measured in the low Ce-doped glass standard (Istd) under the same HV condition is called the $k$-ratio. For each high voltage used, mean $k$-ratio is calculated over more than one hundred measurements. Mass thickness and Ce content of the top layer are then computed by the TFA program to get the best fit of the experimental k-ratios obtained at 8 and $15 \mathrm{kV}$ (Figure 3). Thus, a $50 \mathrm{~nm}$ depth layer containing $1200 \mathrm{ppmw}$ of cerium is the best solution.

Consequently, the EPMA analysis confirms that the cerium is embedded in the extreme surface and quantifies the concentration around 1200 ppmw.

\subsection{3 $\mid$ XPS results}

XPS technique was not sensitive to the iron contamination in the polished surface layer, nevertheless it could detect the cerium contamination. XPS spectra showing the core level $\mathrm{Ce}_{3 \mathrm{~d}}$ binding energy are shown in Figure 4A. The

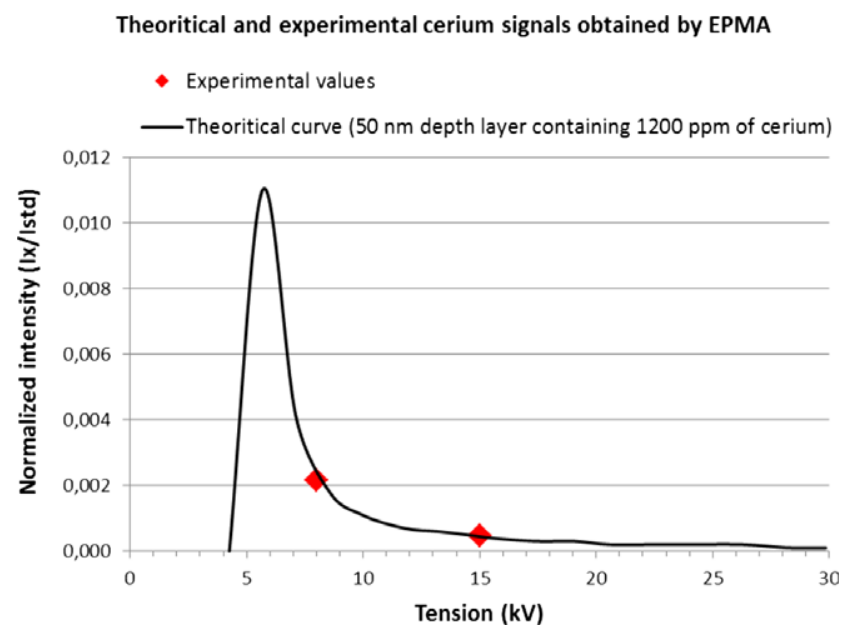

F I G U RE 3 Microprobe best fit between the experimental cerium signals obtained at 8 and $15 \mathrm{kV}$ and the theoretical curve with the Thin Film Analysis mode [Color figure can be viewed at wileyonlinelibrary.com] 

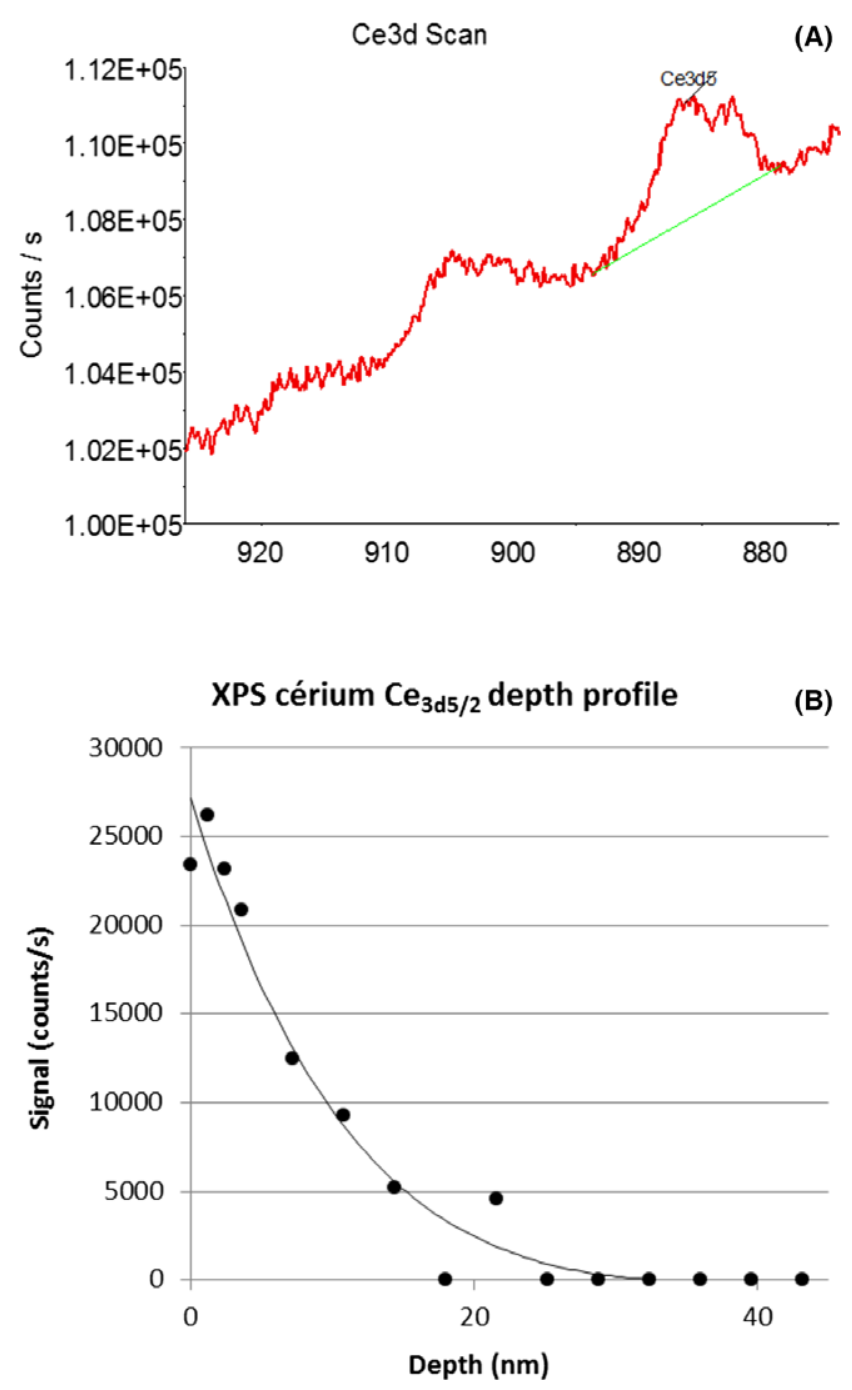

F I G URE 4 XPS measurements on a MRF polished fused silica sample. (A) Ce3d XPS spectra at the surface. (B) XPS depth profile of cerium $\mathrm{Ce}_{3 \mathrm{~d} 5 / 2}$ signal [Color figure can be viewed at wileyonlinelibrary.com]

depth profile of this cerium signal is presented in Figure $4 \mathrm{~B}$.

As mentioned by Juskevicius et al., ${ }^{23}$ the intensity of XPS signal was very low which means that the cerium concentration is very low. Because of this very small signal, we could not quantify the cerium contamination and we chose to plot the signal in counts per second (Figure 4B) instead of the estimated concentration in atomic percent. In this graph, signal presents an exponential decay until about $15 \mathrm{~nm}$. Signal is significant if it is higher than 5000 counts/s because measurements deeper than $20 \mathrm{~nm}$ oscillated between 0 and 5000 count/s. This pollution penetration depth obtained by XPS is typically 2.5-3 times smaller than the ones obtained by SIMS and EPMA measurements, but it can be explained by the poor sensitivity of the XPS technique for these low concentrations. Indeed in XPS analysis, the noise level was reached as soon as the maximal intensity was divided by only 5, whereas in SIMS the noise level was much lower and was reached when the maximal intensity was divided by 200 . Nevertheless, the signal decay is very similar for both analyses. SIMS analysis is more sensitive and so can detect cerium in deeper layers. To emphasize this comparison, we can notice that for both analyses the maximal intensity is divided by 5 at $20 \mathrm{~nm}$ depth.

\subsection{4 | ICP-OES results}

SIMS, XPS, and EPMA analyses tend to confirm a 60-nmdeep surface layer containing 1200 ppmw of cerium. However, these measurements were performed on very small areas of few hundreds of $\mu \mathrm{m}^{2}$. On the other hand, ICPOES measurements allow us to probe the whole sample surface. Consequently, we performed ICP-OES experiments on a MRF polished fused silica sample to get complementary information. The sample was etched in a $\mathrm{HF} / \mathrm{HNO}_{3}$ solution and the thicknesses of removed layers were measured by mass losses using the protocol described in Section 2.7. Figure 5 illustrates the cerium concentration depth profile obtained.

As previously noticed by Neauport et al. ${ }^{7}$ and Catrin et al., ${ }^{14}$ the cerium concentration decreases with depth but is still significant after a few micrometers below the surface. Regarding cerium concentration on the surface, we measured the same order of magnitude as Neauport et al. ${ }^{7}$ and Gao et al., ${ }^{13}$ ie, hundreds of ppmw on a surface layer of $\approx 1 \mu \mathrm{m}$. However, this quantity is not consistent with our EPMA measurement $(\approx 1200 \mathrm{ppmw})$ and Suratwala's results. ${ }^{4}$ And even if it is known that different polishing processes can explain a large distribution of impurities concentration at the extreme surface, ${ }^{4,13}$ the samples

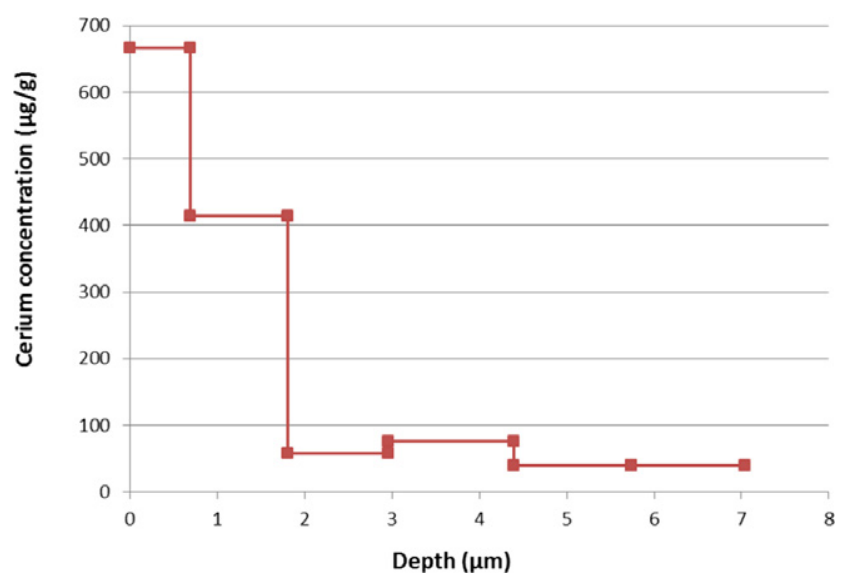

F I G U RE 5 Cerium concentration depth profile measured by ICP-OES on a MRF polished sample [Color figure can be viewed at wileyonlinelibrary.com] 


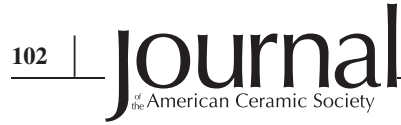

characterized by EPMA and ICP-OES measurements in this study were polished under the same conditions and so the two techniques should give the same results. Consequently, the large discrepancy between the ICP-OES results and the ones retrieved from the other spectroscopic techniques presented in this study was proposed to be due to the heterogeneity of the samples. Indeed, we proposed that most of the pollution could be aggregated at the surface on localized "island" as proposed by Liao et al. ${ }^{24}$ or embedded deeper in scratches or concentrated on chamfer. To address this hypothesis, we performed surface mapping using SEM, SIMS, and EPMA.

\subsection{Surface mapping and homogeneity analysis}

\subsection{1 | SEM characterization}

Liao et al. ${ }^{24}$ demonstrated significant spatial variations in the surface composition at a scale length of several micrometers using a SEM Back Scattered Electrons (BSE) observation. This composition variation was attributed to cerium isolated islands induced by the polishing process. We performed similar SEM image in BSE mode which is sensitive to chemical composition instead of topology. No cerium islands have been detected at the surface. In contrast with Liao et al., ${ }^{24}$ the surface composition appeared to be uniform. This result is consistent with the investigation conducted by Suratwala et al. ${ }^{4}$ but cannot explain the ICPOES results.

\subsection{2 | SIMS analysis around a scratch}

SEM observations showed that pollution is not agglomerated at the surface. Consequently, we focused on our second hypothesis that the contamination is embedded deeper in surface defects such as scratches. Indeed, studies $^{25,26}$ present generally the subsurface damage and contamination with a schematic representation as presented in Figure 6. In this representation, scratches are specific places for the polishing-induced pollution.

To confirm this schematic illustration and to provide a more thorough analysis of the contamination distribution on the polished fused silica surface, we performed a SIMS analysis around a scratch which width was $40 \mu \mathrm{m}$ after MRF polishing. Figure 7A is an optical micrograph observation that illustrates the scratch and the location of two depth profiles measurements, ie, in the scratch and on the pristine surface next to the scratch. Before abrasion, we revealed the spatial distribution of the cerium and iron signals on the analysis area (cf Figure 7B,C) expressed in number of counts. Signals are maxima in the scratch so this spatial analysis confirms that the pollutants are concentrated in the scratch.

To complete this SIMS analysis, we performed the cerium and iron concentration depth profiles on the scratch and on the pristine surface (see Figure 7A). Measurements in the scratch are represented with a continuous line in Figure 8 and measurements out of the scratch with a dashed line.

The contamination depth profiles exhibit an exponential decay until $800 \mathrm{~nm}$ in the scratch for cerium (Figure 8A) and around $300 \mathrm{~nm}$ for iron (Figure $8 \mathrm{~B}$ ). The profiles related to the point located next to the scratch, corresponding to the pristine surface, reveal a contamination penetration similar to the one retrieved from the other SIMS measurements presented in the first part of this study, namely, $60 \mathrm{~nm}$ for the cerium and $30 \mathrm{~nm}$ for the iron. Consequently, contaminants are mostly concentrated in the scratch where they penetrate deeper than in other locations of the surface without defects.

To conclude, this SIMS analysis reveals a nonhomogeneous repartition of the impurities and demonstrates that scratches are preferential sites for the polishing-induced contamination. However, scratches are not sufficient to explain the deeper pollutants penetration measured by ICP-OES.

As explained in Section 2.7, ICP-OES technique uses an acid that leaches not only the surface but also the

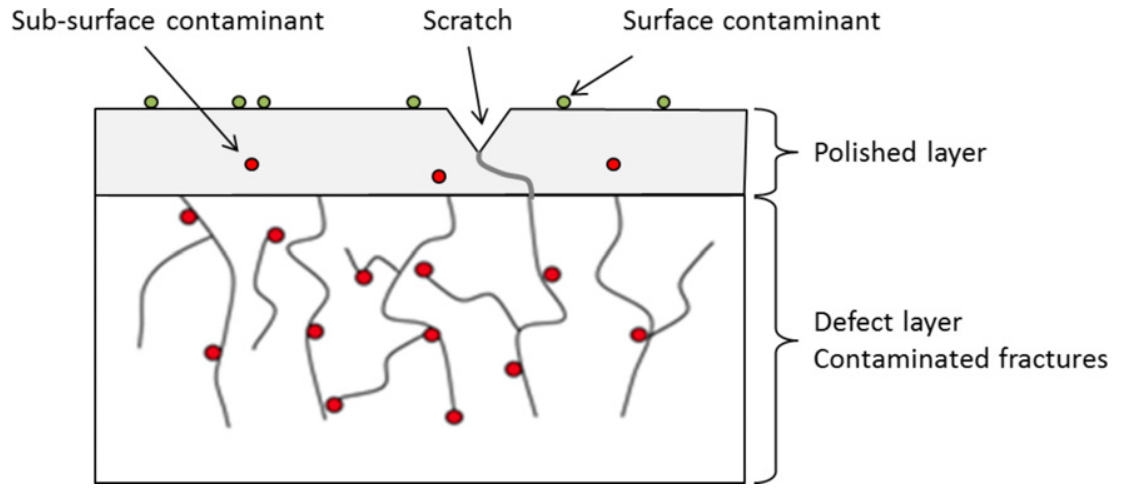

F I G U RE 6 Schematic illustration of the subsurface defects and contaminants on the polished fused silica surface [Color figure can be viewed at wileyonlinelibrary.com] 


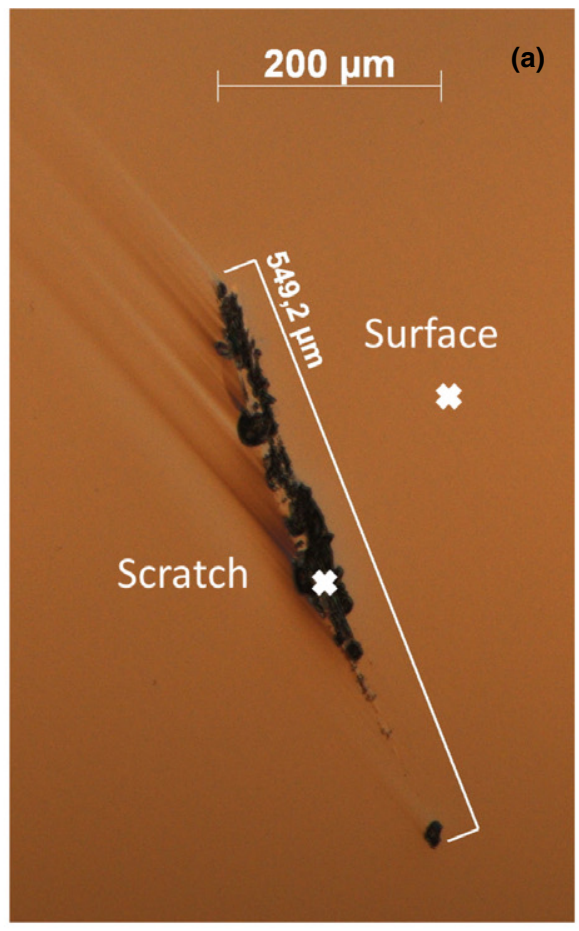

(b)

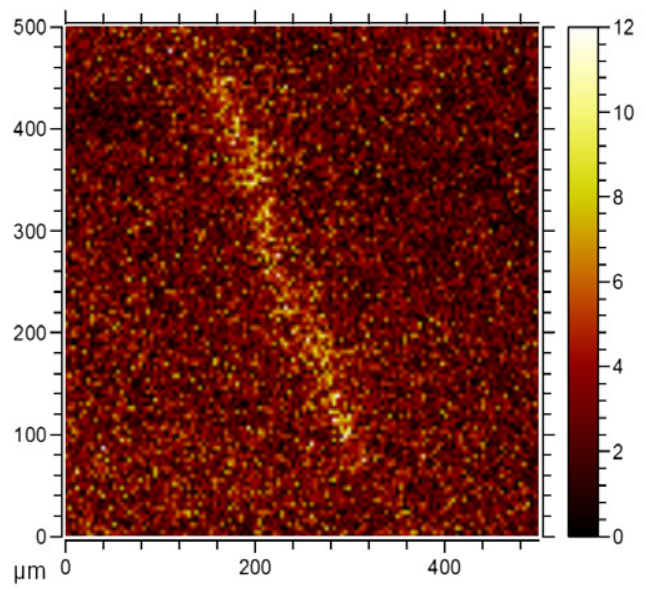

(c)

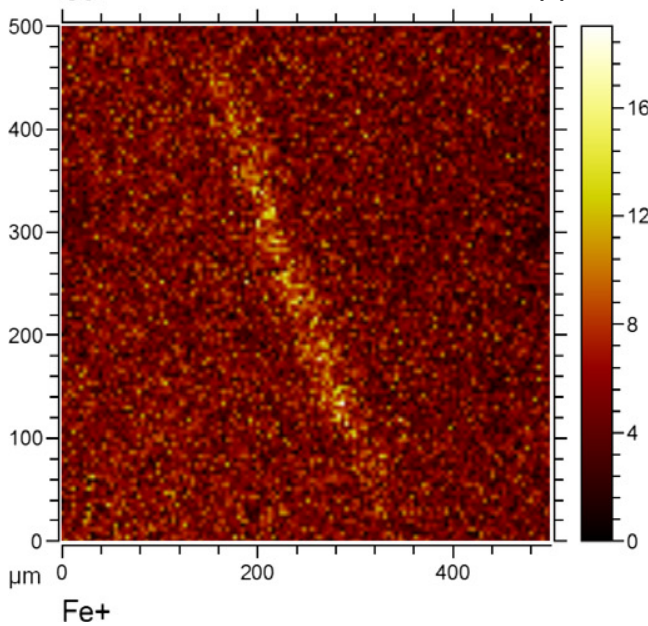

F I G URE 7 (A) Optical micrograph observation of a scratch. Surface mapping of the cerium (B) and iron (C) signals before abrasion, measured by SIMS. [Color figure can be viewed at wileyonlinelibrary.com]
Cerium depth penetration

(A)

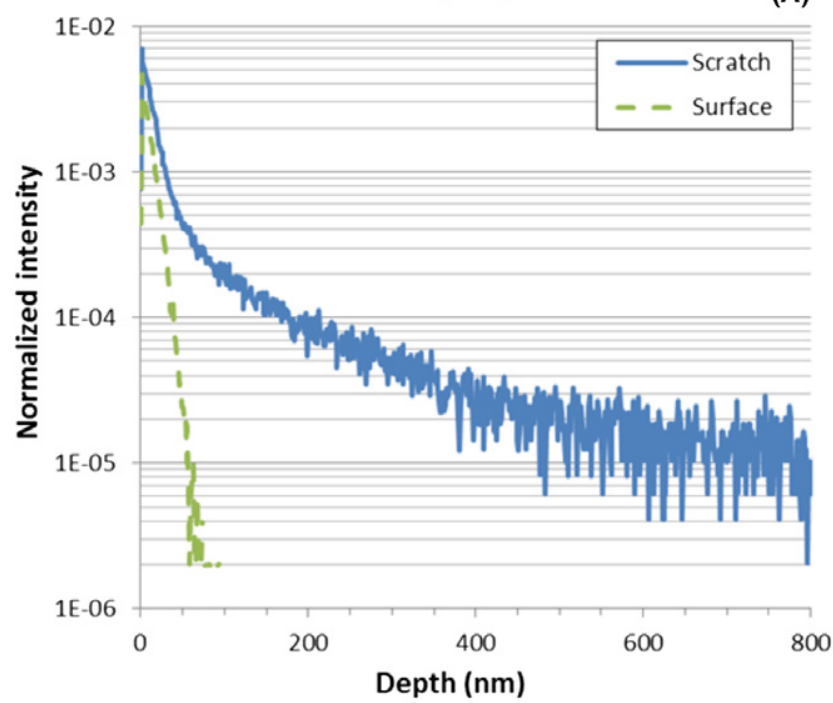

Iron depth penetration

(B)

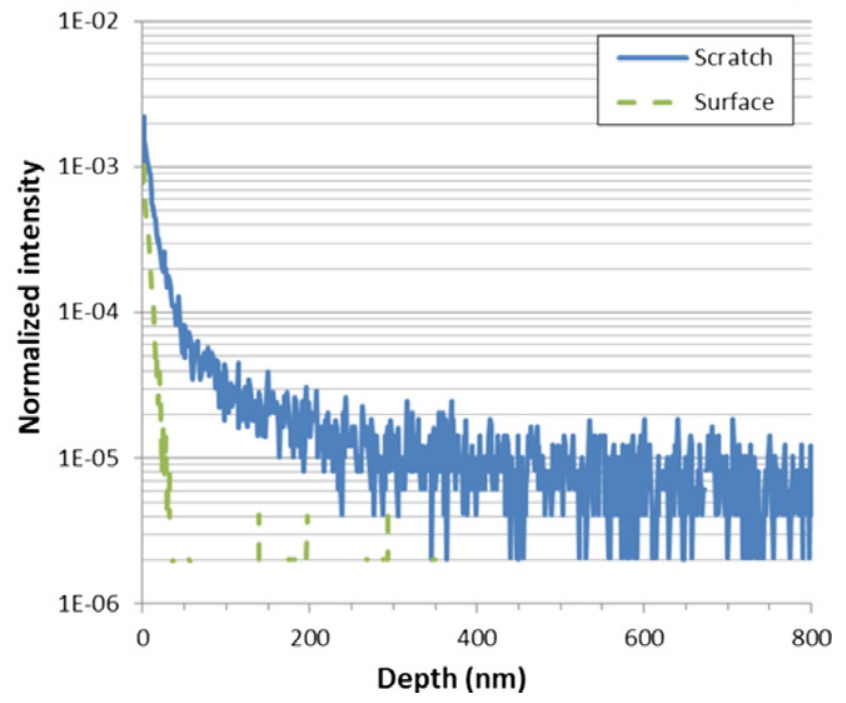

F I G URE 8 Cerium (A) and iron (B) SIMS depth profiles in a scratch (blue plain line) and at the surface (green dashed line) [Color figure can be viewed at wileyonlinelibrary.com]

chamfer. So it can bias the results. Thus, we measured the polish-induced contamination in the chamfer of the samples to complete our contamination repartition analysis.

\subsection{3 | EPMA analysis on the chamfer}

Microprobe was the first technique used to analyze the pollution in the chamfer. A line of 120 measurements of cerium signal was carried out at $8 \mathrm{kV}$ at the interface between the surface and bevel (Figure 9). Points of measurements were spaced out with a distance of $10 \mu \mathrm{m}$. This analysis was not quantitative but only provided relative data of the intensity of the cerium signal between the chamfer and the surface. 


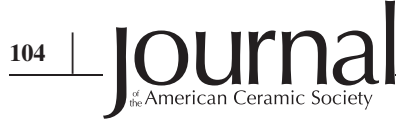

Cerium signal in the chamfer and on the surface

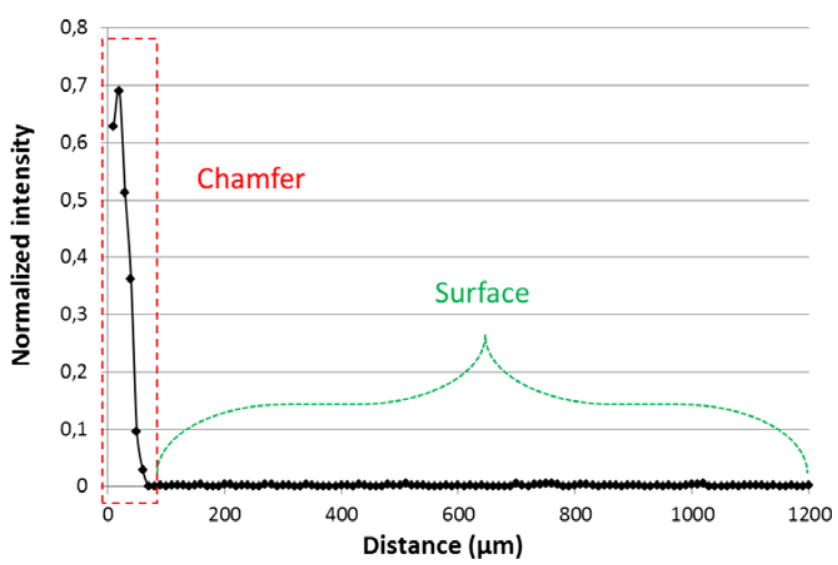

F I G URE 9 Cerium signal measurements by EPMA at the interface between surface and chamfer [Color figure can be viewed at wileyonlinelibrary.com]

The intensity of cerium signal was much more important in the bevel so EPMA has revealed an excessive cerium contamination on the chamfer.

$\mathrm{X}$-ray mapping of cerium and iron contamination presented in Figure 10 shows that iron is also in excess in the chamfer and agglomerates as particles, whereas cerium is spread more homogeneously. The cerium signal decreases at the left of the analysis area, it might be due to the $45^{\circ}$ tilt between the bevel and the electron beam which is not an optimum measurement condition.

Grinding and polishing steps prior final finishing with MRF were done with alumina and conventional $\mathrm{CeO}_{2}$ polishing fluid, respectively. Consequently, the iron contamination is only from MRF polishing. During this process, chamfer is deeply dived in the MRF ribbon. Moreover, chamfers are grinded whereas the surface is polished; so the pollution is probably easily embedded in the chamfer than in the surface.

\subsection{4 | Additional SIMS analysis on the chamfer}

Cerium and iron contaminations were investigated by SIMS on three spots located in the chamfer: one close to the surface, one close to the vertical section, and one in the middle of the chamfer. Cerium and iron depth profiles obtained are represented in Figure 11.

Previous SIMS measurements performed at the surface and presented in the Section 3.2.1 revealed a cerium penetration until $60 \mathrm{~nm}$ deep under the surface and $30 \mathrm{~nm}$ deep for iron. Measures on the chamfer, presented Figure 11, showed that cerium and iron signals are still significant $800 \mathrm{~nm}$ under the surface. Consequently, it appeared that cerium and iron pollutions decreased much more slowly in the chamfer than on the polished surface. We can also
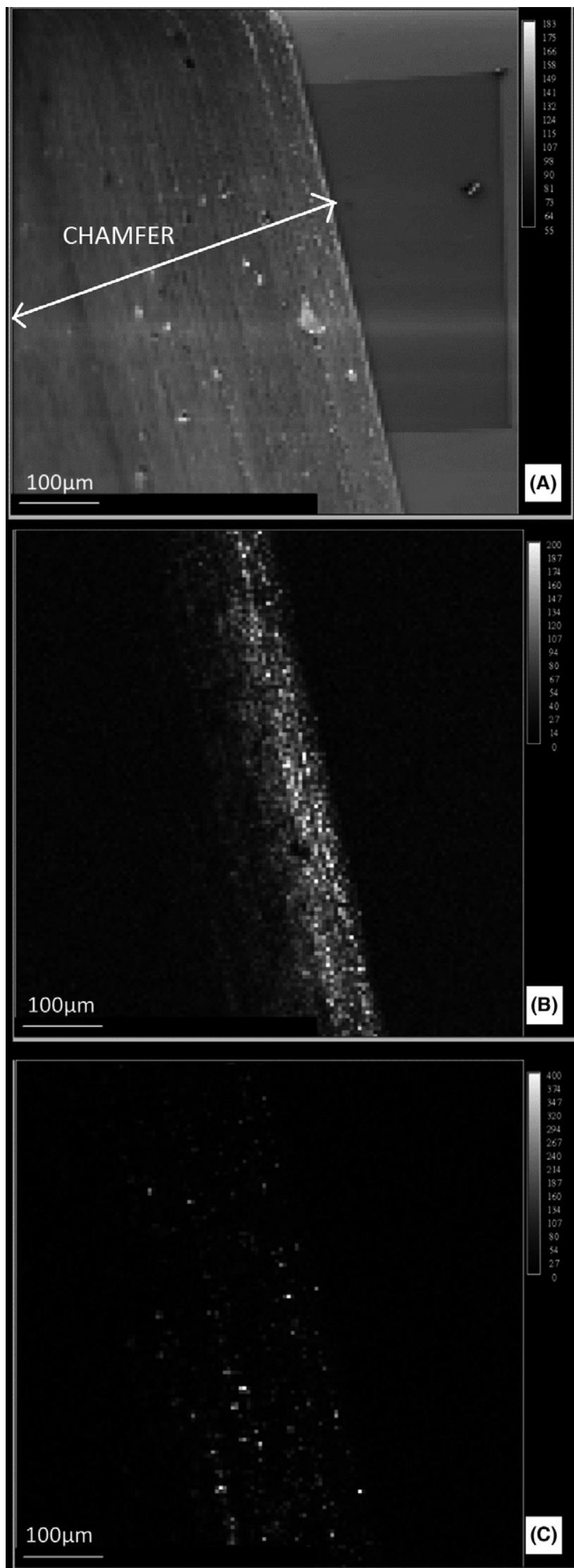

F I G U RE 10 Impurities mapping obtained by EPMA at the interface between the surface and the chamfer: SEM observation of the area analyzed (A), cerium signal (B) and iron signal (C) 

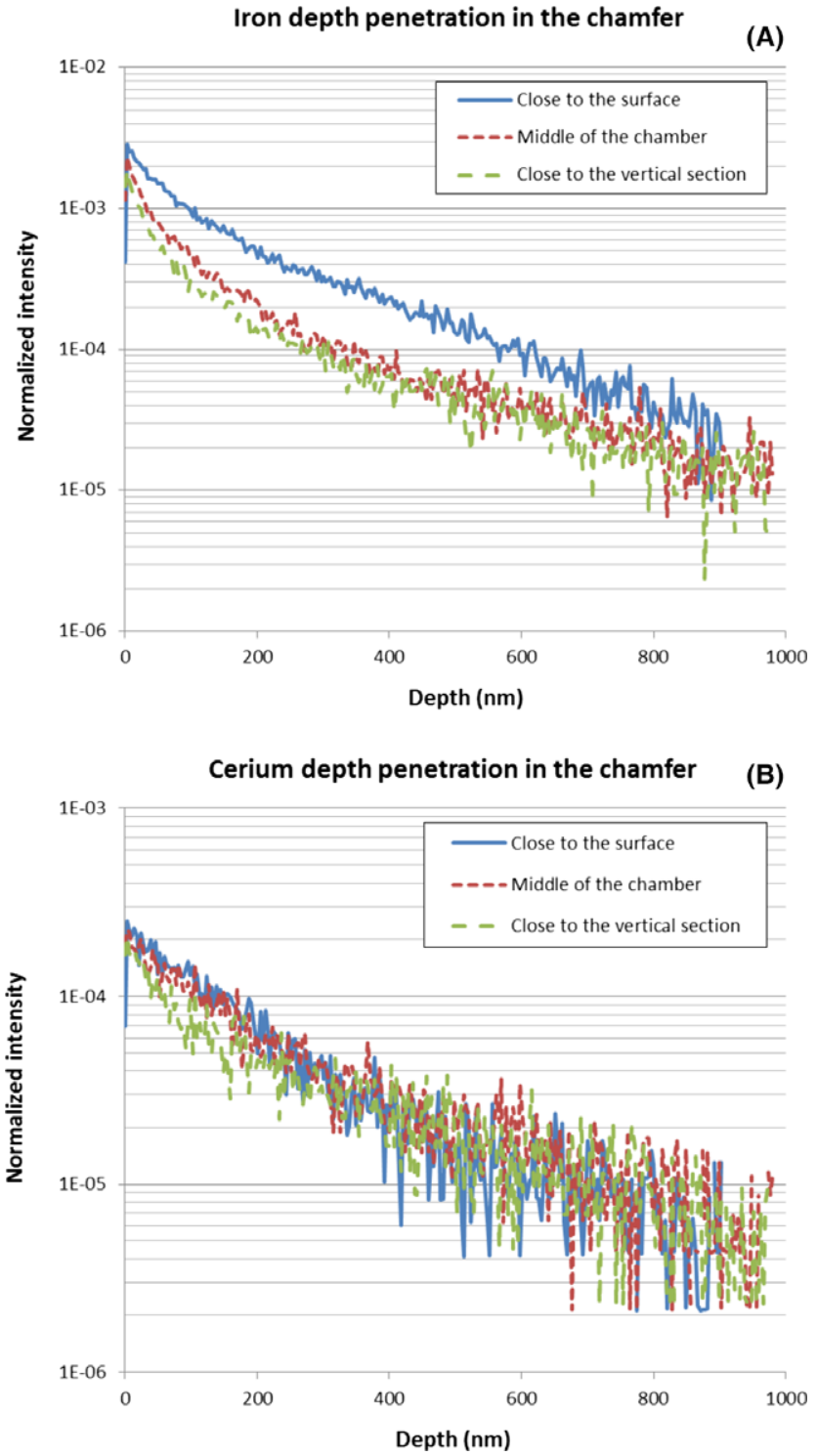

F I G U RE 11 Iron (A) and Cerium (B) SIMS depth profiles in the chamfer [Color figure can be viewed at wileyonlinelibrary.com]

notice that the cerium signals (b) are identical for the three plots, whereas iron signals depend on the spot location. This is an indicator that cerium pollution is homogenous in the chamfer, whereas iron is not. The iron signal mapping obtained for the spot measurement located in the chamfer close to the surface reveals an iron agglomeration with an approximated size around 5-10 $\mu \mathrm{m}$ (Figure 12B). On the opposite cerium signal is homogeneous on the entire analysis area (Figure 12A).

This observation is coherent with the EPMA mapping on the chamfer presented in Section 3.3.3. The iron spatial distribution in the chamfer can be related to the TEM polishing fluid observations presented in Section 2.2 which shows that iron particles were bigger than cerium particles. Because of the roughness of the chamfer, agglomerate of iron particles can be embedded in the surface of the chamfer.

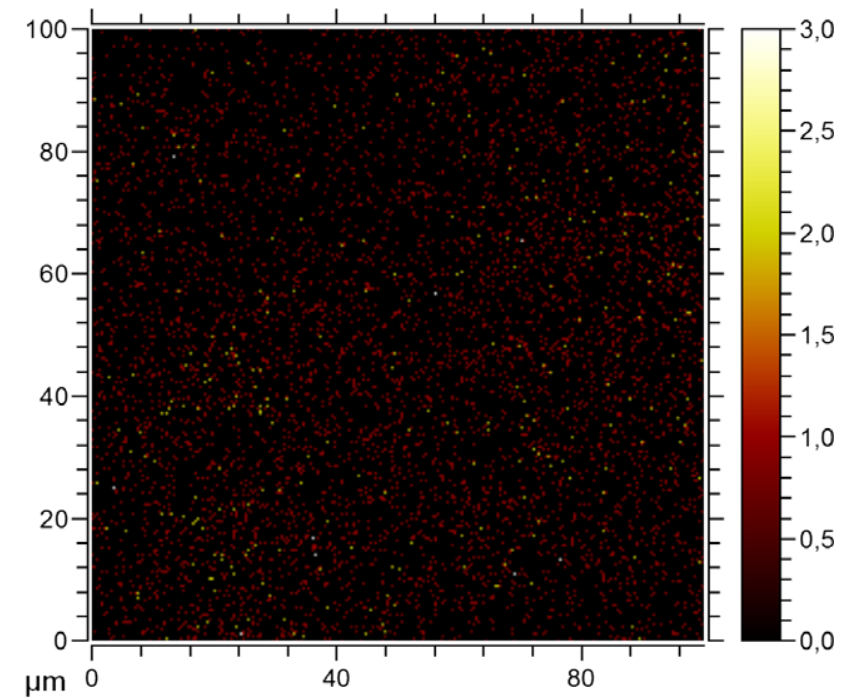

(A) $\mathrm{Ce}+$

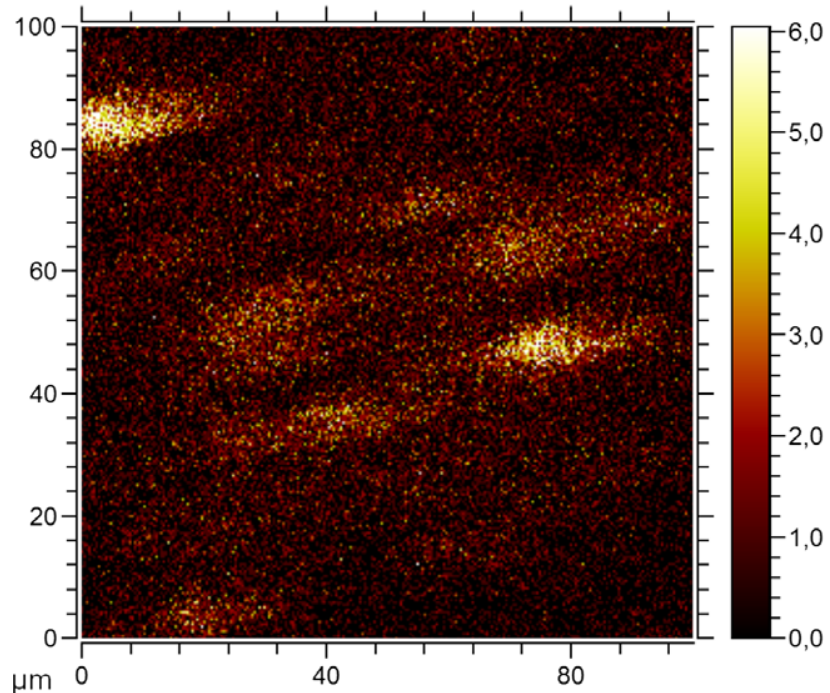

(B) $\mathrm{Fe}+$

F IG URE 12 Cerium (A) and iron (B) signals mapping in the chamfer before abrasion, measured by SIMS [Color figure can be viewed at wileyonlinelibrary.com]

\subsection{5 | ICP-OES on a sample without chamfer}

To confirm our assumption that the ICP-OES technique measures a deeper and higher contamination because it is the only technique which includes scratches and chamfer, we cut a MRF polished fused silica sample to remove the chamfer and we measured cerium contamination by ICPOES. The analysis layers thicknesses were reduced because we expected an asymptotic behavior from the first micrometer under the surface. The cerium depth profile of the sample without chamfer is shown in Figure 13. This profile has to be compared with the profile obtained on the entire sample, including chamfer and scratches, similar to the one 


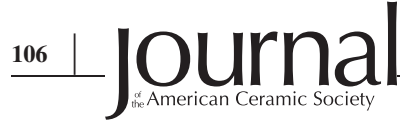

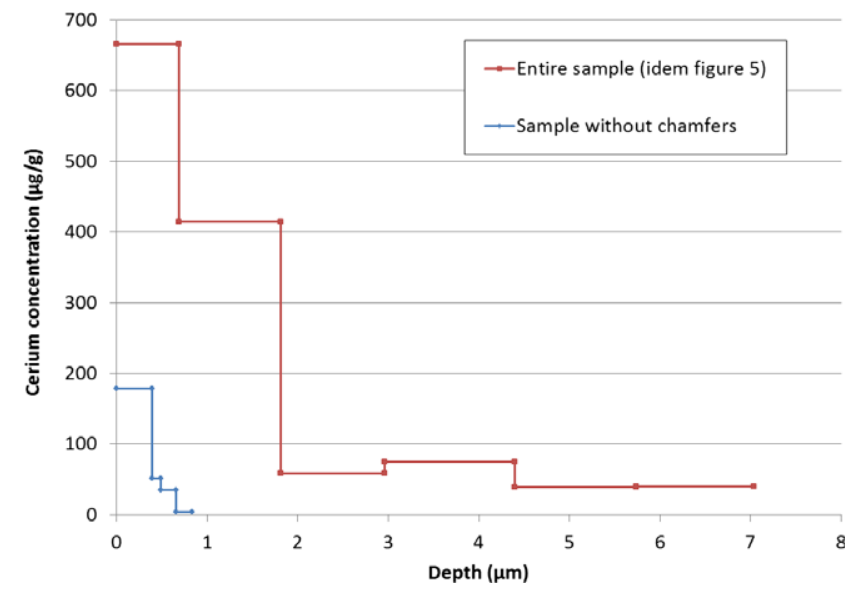

F I GURE 13 Depth profiles of cerium concentration measured by ICP-OES on a MRF polished sample (red) and on a MRF polished sample without chamfer (blue) [Color figure can be viewed at

wileyonlinelibrary.com]

presented in Figure 5 and reproduced in Figure 13 for comparison purpose.

Without chamfer the cerium concentration falls down before the first micrometer. This measurement proves that the overconcentration on chamfers is the main reason why ICP-OES overestimates the impurities penetration until a few micrometers.

\section{4 | CONCLUSIONS}

Surface characterization of silica samples has been carried out with various selected analytical techniques: ToF-SIMS, EPMA, XPS, and ICP-OES. These techniques were used to further study the pollution induced by polishing and it should be emphasized that none of these techniques could on their own provide a complete description. SIMS was found to be the most sensitive technique because it is the only one which detected the iron contamination. SIMS was also useful to precisely measure depth profiles, but the small analysis area could not permit to discuss about the possible pollution heterogeneity. SIMS can be used to quantify the pollution but a complicated calibration has to be done prior to measurement. EPMA was found to be perfectly adapted to map the surface and the TFA mode permitted to quantify the cerium concentration in the surface layer. XPS was found to be not sensitive enough to measure such small concentrations, although it was a good technique to study the extreme surface. Finally, ICP-OES was the only technique that allowed us to consider the entire surface sample, but the mandatory sample preparation potentially induces an important risk of biasing the results. All the techniques presented in this study offered complementary information on the interface composition and illustrated the specific role of cerium and iron.

This work confirms that the fused silica polished surface contamination is composed of alkali and metallic elements from the polishing fluid. SIMS revealed that iron penetrates on $30 \mathrm{~nm}$ under the surface, whereas cerium is present in a 60-nm-deep surface layer. EPMA confirmed the cerium depth penetration and estimated the cerium concentration at 1200 ppmw. SIMS indicated that contamination is nonhomogeneous and surface defects such as scratches are specific places which hold the pollutants. Iron and cerium concentrations are higher in scratches where these impurities go deeper than in the surface.

These cerium and iron overconcentrations have also been observed in the chamfer.

This new insight about the impurities spatial distribution into the bevel does not influence the laser damage threshold for optical components because the chamfer is not a laser crossing area. However, it was useful to explain the disparities between the results obtained with ICP-OES on the one hand and SIMS, EPMA, and XPS on the other hand.

\section{ACKNOWLEDGMENTS}

We would like to gratefully acknowledge the help of Biophy Research $^{27}$ and especially Laurent Dupuy for SIMS measurements, the help of Placamat ${ }^{28}$ platform for XPS measurement, and our CEA colleagues for EPMA and ICPOES measurements. This work benefited from the support of the project LAPHIA ANR-10-IDEX-03-02 of the French National Research Agency (ANR).

\section{REFERENCES}

1. Beilby SG. Aggregation and flow of solids. London: Macmillan; 1921.

2. Trogolo JA, Rajan K. Near surface modification of silica structure induced by chemical/mechanical polishing. J Mater Sci. 1994;29:4554-4558.

3. Bach H. Analysis of surface layers. SPIE. 1983;381:113-128.

4. Suratwala T, Steele W, Wong L, Feit M, Miller P, et al. Chemistry and formation of the Beilby layer during polishing of fused silica glass. J Am Ceram Soc. 2015;98.

5. Bach H. Advanced surface analysis of silicate glasses, oxides and other insulating materials: A review. J Non-Cryst Solids. 1997;209:1-18.

6. Kozlowski M, Carr J, Hutcheon I, Torres R, Sheehan L, et al. Depth profiling of polishing-induced contamination on fused silica surfaces. Laser Induced damage in optical materials: 1997. Proc. SPIE. 1998;3244:365375.

7. Neauport J, Lamaignere L, Bercegol H, Pilon F, Birolleau JC. Polishinginduced contamination of fused silica optics and laser induced damage density at $351 \mathrm{~nm}$. Opt Express. 2005;13:10163-10171.

8. Bloembergen N. Role of cracks, pores, and absorbing inclusions on laserinduced damage threshold at surfaces of transparent dielectrics. Appl Opt. 1973;12:661-664.

9. Camp DW, Kozlowski MR, Sheehan LM, Nichols M, Dovik M, et al. Subsurface damage and polishing compound affect the 355-nm laser 
damage threshold of fused silica surfaces. Laser-induced Damage in Optical Materials: 1997. Proc. SPIE. 1998;3244:356-364.

10. Miller PE, Suratwala TI, Bude JD, Laurence TA, Shen N, et al. Identification of laser damage precursors in fused silica. Laser-induced Damage in Optical Materials: 2009. Proc. SPIE. 2009;7504.

11. Liu H, Ye X, Zhou X, Huang J, Wang F, et al. Subsurface defects characterization and laser damage performance of fused silica optics during HFetched process. Opt Mater. 2013;36:855-860.

12. Jiang X, Liu Y, Rao H, Fu S. Improve the laser damage resistance of fused silica by wet surface cleaning and optimized HF etch process. Pacific Rim Laser Damage 2013: Optical Materials for High Power Lasers. Proc SPIE. 2013;8786.

13. Gao X, Feng G, Zhai L, Shouhuan Z. Effect of subsurface impurities of fused silica on laser-induced damage probability. Opt Eng. 2014;53.

14. Catrin R, Neauport J, Taroux D, Cormont P, Maunier C, et al. Magnetorheological finishing for removing surface and subsurface defects of fused silica optics. Opt Eng. 2014;53.

15. Wang Z, Wang L, Yang J, Peng W, Hu H. Detection of subsurface trace impurity in polished fused silica with biological method. Opt Express. 2014;22:21292-21301.

16. Wang Z, Wang L, Peng W, Cao Y, Yang J, et al. Origin and distribution of redeposition layer in polished fused silica. Opt Eng. 2015;54.

17. Wang J, Li Y, Yuan Z, Ye H, Xie R. Producing fused silica optics with high UV-damage resistance to nanosecond pulsed lasers. Pacific Rim Laser Damage 2015: Optical Materials for High-Power Lasers. Proc. SPIE. 2015;9532.

18. Ye H, Li Y, Yuan Z, Wang J, Xu Q. Improving UV laser damage threshold of fused silica optics by wet chemical etching technique. Pacific Rim Laser Damage 2015: Optical Materials for High-Power Lasers. Proc. SPIE. 2015;9532.

19. Suzuki S, Kakita K. A comparative study of GDOES, SIMS and XPS depth profiling of thin layers on metallic materials. Journal of surface analysis. 2005;12:174-177.

20. Shimizu K, Habazaki H, Skeldon P, Thompson G. Radiofrequency GDOES a powerful technique for depth profiling analysis of thin films. Surf Interface Anal. 2003;35:564-574.
21. Golini D, Kordonski W, Dumas P, Hogan S. Magnetorheological finishing (MRF) in commercial precision optics manufacturing. Optical Manufacturing and Testing. Proc. SPIE. 1999;3782:80-91.

22. Pouchou J. X-ray microanalysis of thin surface films and coatings. Mikrochim Acta. 2002;138:133-152.

23. Juskevicius K, Buzelis R, Kicas S, Tolenis T, Drazdys R, et al. Investigation of subsurface damage impact on resistance of laser radiation of fused silica substrates. Laser-Induced damage in optical materials: 2013. Proc. SPIE. 2013;8885.

24. Liao D, Chen X, Tang C, Xie R, Zhang Z. Characteristics of hydrolyzed layer and contamination on fused silica induced during polishing. Ceram Int. 2014;40:4479-4483.

25. Battersby CL, Sheehan LM, Kozlowski MR. Effects of wet etch processing on laser-induced damage of fused silica surfaces. Laserinduced Damage in Optical Materials: 1998. Proc. SPIE. 1998;3578:446455.

26. Suratwala TI, Miller PE, Bude JD, Steele WA, Shen N. HF-based etching processes for improving laser damage resistance of fused silica optical surfaces. J Am Ceram Soc. 2010;94:416-428.

27. BIOPHY-RESEARCH, ZAC Saint Charles, 131 avenue de l'étoile, 13710 Fuveau, France. www.biophyresearch.com.

28. PLACAMAT, 87 avenue du docteur Albert Schweitzer, 33608 Pessac Cedex, France.

How to cite this article: Pfiffer M, Longuet J-L, Labrugère $\mathrm{C}$, et al. Characterization of the polishinginduced contamination of fused silica optics. $J \mathrm{Am}$ Ceram Soc. 2017;100:96-107. doi:10.1111/jace.14448. 